\title{
EXERCÍCIO FÍSICO E FATORES DE RISCO CARDIOVASCULARES EM MULHERES OBESAS NA PÓS-MENOPAUSA
}

\author{
PHYSICAL EXERCISE AND CARDIOVASCULAR RISK FACTORS IN OBESE WOMEN IN \\ THE POSTMENOPAUSAL PERIOD
}

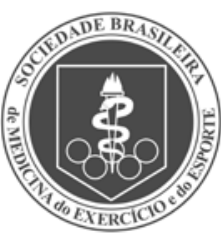

Artigo Original

\section{EJERCICIO FÍSICO Y FACTORES DE RIESGO CARDIOVASCULARES EN MUJERES OBESAS EN LA POST MENOPAUSIA}

\author{
Eduardo Federighi Baisi Chagas ${ }^{1-3}$ \\ (Educador Físico) \\ Mariana Rotta Bonfim² \\ (Educador Físico) \\ Nair Cristina Magarido Brondino 5 \\ (Estatística) \\ Henrique Luiz Monteiro 2,4 \\ (Educador Físico)
}

1. Universidade de Marília (UNIMAR), Marília, SP, Brasil.

2. Laboratório de Avaliação e Prescrição de Atividade Física (LAPE), Bauru, SP, Brasil.

3. Faculdade de Ciências e

Tecnologia do Campus de

Presidente Prudente (UNESP),

Presidente Prudente, SP, Brasil.

4. Faculdade de Ciências,

Departamento de Educação Física/

UNESP, Bauru, SP, Brasil.

5. Faculdade de Ciências,

Departamento Matemática/ UNESPBauru, SP, Brasil.

\section{Correspondência:}

Universidade de Marília, Departamento de Educação Física. Av. Higino Muzzy Filho, 1001, Jardim Universitário, Marília-SP. 17.000-000. efbchagas@hotmail.com

\section{RESUMO}

Introdução: o exercício físico tem sido recomendado, como estratégia terapêutica não farmacológica, no tratamento de importantes fatores de risco cardiovascular. Objetivo: analisar o impacto de um programa de exercício físico, adaptado à realidade das Unidades de Saúde da Família (USF), sobre a composição corporal, fatores de risco cardiovasculares e Escore de Framingham de mulheres obesas na pós-menopausa. Métodos: 70 mulheres entre 50 e 79 anos, sedentárias, obesas e sem menstruar por no mínimo doze meses, foram distribuídas aleatoriamente em um grupo treinado (GT) $(n=35)$ e um não treinado ( $G n T)(n=35)$. O GT participou de vinte semanas de um programa de exercícios físicos com três sessões semanais, constituído por atividades de monitoramento e aquecimento (10 min); 25 min de exercício de flexibilidade e força; 50 min de caminhada com intensidade entre 50 a $65 \%$ do $\mathrm{VO}_{2 \text { maxi }}$ e 5 min de relaxamento. $\mathrm{O} \mathrm{GnT}$ foi orientado a manter suas atividades normais. Resultados: o GT apresentou reduções significativas do índice de massa corporal (30,1+3,7 vs. 29,3+3,7; $p=0,0001)$, circunferência de cintura $(93,3+10,3$ vs. $89,1+10,4 ; p=0,0001)$, percentual de gordura $(54,2+2,9$ vs. $53,2+3,3 ; p=0,0001)$, pressão arterial sistólica $(128,0+14,6$ vs. 119,2+10,3; $p=0,0001)$, triglicerídeos $(148,4+66,1$ vs. $122,8+40,7 ; p=0,006)$, VLDL colesterol $(29,7+13,2$ vs. $24,5+8,0 ; p=0,005)$ e Escore de Framingham $(13,08+4,0$ vs. $11,77+4,1 ; p=0,010)$. No grupo não treinado foram observados aumentos significativos do percentual de gordura $(55,0+4,0$ vs. $57,0+3,8 ; p=0,0001)$, pressão arterial sistólica $(128,6+10,5$ vs. $133,7+12,0 ; p=0,001)$, glicemia de jejum $(95,2+18,4$ vs. $113,7+28,8 ; p=0,001)$ e Escore de Framingham (12,82+3,2 vs. 13,91+4,0; $p=0,043)$, como também reduções do HDL colesterol $(55,1+10,5$ vs. 51,7+11,0; $p=0,017)$. Conclusão: o programa de exercício físico adaptado às condições de USF foi efetivo na redução dos fatores de risco cardiovascular de mulheres obesas na pós-menopausa atendidas pelo programa SUS.

Palavras-chave: mulheres, pós-menopausa, obesidade, fatores de risco, doenças cardiovasculares.

\section{ABSTRACT}

Introduction: physical exercise has been recommended as a non-pharmacological, therapeutic strategy in the treatment of important cardiovascular risk factors. Objective: to analyze the impact of an exercise program, tailored to the reality of the Family Health Units (FHU), on body composition, cardiovascular risk factors and Framingham score in obese postmenopausal. Methods: 70 women between 50 and 79 years, sedentary, obese and without menstruating for at least twelve months, were randomly assigned to a trained group (TG) $(n=35)$ and an untrained (GnT) (n=35). The GT took 20 weeks of a physical exercise program with three weekly sessions, consisting of monitoring activities and heating (10 minutes), 25 minutes of exercise flexibility and strength, 50 minute walk with intensity between 50$65 \%$ of VO2max and 5-minute cool-down. The GnT was instructed to maintain their normal activities. Results: TG showed significant reductions in body mass index $(30,1+3,7 \mathrm{vs}$. 29,3+3,7; $p=0,0001)$, waist circumference $(93,3+10,3$ vs. $89,1+10,4 ; p=0,0001)$, percentage of fat $(54,2+2,9$ vs. $53,2+3,3 ; p=0,0001)$, systolic blood pressure $(128,0+14,6$ vs. $119,2+10,3 ; p=0,0001)$, triglycerides $(148,4+66,1$ vs. $122,8+40,7 ; p=0,006), V L D L$ cholesterol $(29,7+13,2$ vs. $24,5+8,0$; $p=0,005)$ and Framingham score $(13,08+4,0 \mathrm{vs} .11,77+4,1 ; p=0,010)$. In the untrained group were observed significant increases in the percentage of fat $(55,0+4,0 \mathrm{vs.} 57,0+3,8 ; p=0,0001)$, systolic blood pressure $(128,6+10,5 \mathrm{vs}$. $133,7+12,0 ; p=0,001)$, fasting glucose $(95,2+18,4$ vs. $113,7+28,8 ; p=0,001)$ and Framingham score $(12,82+3,2 \mathrm{vs}$. $13,91+4,0 ; p=0,043)$, but also decreases levels of $H D L$ cholesterol $(55,1+10,5$ vs. $51,7+11,0 ; p=0,017)$. Conclusion: the exercise program, adapted to the conditions of $\mathrm{FHU}$, was effective in reducing cardiovascular risk factors in obese postmenopausal women served by the SUS program.

Keywords: women, postmenopausal, obesity, risk factors, cardiovascular disease.

\section{RESUMEN}

Introducción: El ejercicio físico ha sido recomendado como estrategia terapéutica no farmacológica, en el tratamiento de importantes factores de riesgo cardiovascular. Objetivo: analizar el impacto de un programa de ejercicio fisico adaptado a la realidad de Unidades de Salud de la Familia (USF) sobre la composición corporal, factores de riesgo cardiovasculares y valor de Framingham de mujeres obesas en la post menopausia. Métodos: setenta mujeres entre 50 y 79 años, sedentarias, obesas y sin menstruar por como mínimo doce meses fueron distribuidas aleatoriamente 
en grupo entrenado (GT) ( $n=35)$ y no entrenado (GnT) (n=35). El GT participó de veinte semanas de un programa de ejercicios físicos con tres sesiones semanales, constituido por actividades de monitoreo y calentamiento (10 minutos); 25 minutos de ejercicio de flexibilidad y fuerza; 50 minutos de caminata con intensidad entre 50 a 65\% del VO2max; y 5 minutos de vuelta a la calma. El GnT fue orientado a mantener sus actividades normales. Resultados: el GT presentó reducciones significativas del índice de masa corporal (30,1+3,7 vs.29,3+3,7; $p=0,0001)$, circunferencia de cintura $(93,3+10,3$ vs. $89,1+10,4 ; p=0,0001)$, porcentual de grasa $(54,2+2,9$ vs. 53,2+3,3; $p=0,0001)$, presión arterial sistólica $(128,0+14,6$ vs. 119,2+10,3; $p=0,0001)$, triglicéridos $(148,4+66,1$ vs. 122,8+40,7; $p=0,006), V L D L$ colesterol (29,7+13,2 vs. $24,5+8,0 ; p=0,005)$ y Valor de Framingham $(13,08+4,0 \mathrm{vs} .11,77+4,1 ; p=0,010)$. En el grupo no entrenado se observaron aumentos significativos del porcentual de grasa $(55,0+4,0$ vs. 57,0+3,8; $p=0,0001)$, presión arterial sistólica $(128,6+10,5$ vs. $133,7+12,0 ; p=0,001)$, glicemia de ayuno $(95,2+18,4$ vs. $113,7+28,8 ; p=0,001)$ y Valor de Framingham $(12,82+3,2 \mathrm{vs}$. 13,91+4,0; $p=0,043)$, como también reducciones del HDL colesterol (55,1+10,5 vs. 51,7+11,0; $p=0,017)$. Conclusión: el programa de ejercicio físico adaptado a las condiciones de USF fue efectivo en la reducción de los factores de riesgo cardiovascular de mujeres obesas en la post menopausia atendidas por el programa SUS.

Palabras clave: mujeres, post menopausia, obesidad, factores de riesgo, enfermedades cardiovasculares.

\section{INTRODUÇÃO}

O declínio nos níveis de estrogênios da mulher no período pós-menopausa associa-se a alterações no metabolismo lipídico ${ }^{1,2}$ e potencializa o aumento da adiposidade central e o desenvolvimento da obesidade, ${ }^{3}$ a qual atualmente é identificada como fator de risco cardiovascular independente ${ }^{4}$. Em contrapartida, a prática regular de exercícios físicos é considerada medida terapêutica e preventiva ao desenvolvimento de fatores de risco cardiovasculares, contribuindo para a prevenção e tratamento de doenças crônicas não transmissíveis, bem como para a redução do risco de morte prematura ${ }^{5,6}$.

No Brasil, são recentes as iniciativas quanto à realização de programas de exercício físico em Unidade de Saúde da Família (UBS) e Unidades Básicas de Saúde (USF)7,8, bem como o incentivo do Ministério da Saúde para elevar o nível de atividade física da população ${ }^{9}$. Apesar de estudos demonstrarem efeito significativo do exercício físico sobre fatores de risco cardiovascular ${ }^{6}$, os resultados produzidos até o momento são oriundos, em sua maioria, de pesquisas realizadas em ambientes laboratoriais, que dispõem de recursos tecnológicos e materiais sofisticados, gerando incerteza se programas de exercício físico realizados em ambientes de UBS e USF produziram resultados semelhantes.

Isso se deve pelas limitações de estrutura física e de recursos materiais na realidade de UBS e USF para a realização de programas de exercício físico ${ }^{10}$, sendo necessário dispor de métodos indiretos. Nesse contexto, o objetivo desta pesquisa foi analisar o impacto de um programa de exercício físico adaptado à realidade de Unidades de Saúde da Família (USF) sobre a composição corporal, fatores de risco cardiovasculares e escore de Framingham de mulheres obesas na pós-menopausa.

\section{MATERIAIS E MÉTODOS}

A amostra foi constituída por mulheres na faixa etária de 50 a 79 anos, pós-menopausa (sem menstruação por no mínimo doze meses) ${ }^{2,3}$, obesas (percentual de gordura maior ou igual a 33\%) ${ }^{11}$, previamente sedentárias (menos que 150 minutos por semana de atividade física moderada ou vigorosa, nos últimos seis meses) ${ }^{12}$, todas atendidas na Unidade de Saúde da Família (USF) "Aeroporto" do município de Marília, estado de São Paulo, Brasil. Esta unidade tem 786 mulheres com idade maior ou igual a 50 anos cadastradas, a partir do qual foram selecionadas, de forma aleatória, 140 pacientes para realização das visitas domiciliares e convite a participação do estudo.

Após a visita domiciliar, 94 pacientes responderam positivamente ao convite, porém somente 84 atenderam aos critérios de inclusão, sendo estas alocadas aleatoriamente em dois grupos, a saber: 44 pa- cientes no grupo treinado (GT) e 38 no grupo não treinado (GnT). Ao final do estudo, nove pacientes do grupo treinado e três do grupo não treinado foram excluídas do estudo, por apresentarem dados incompletos, baixa freqüência de participação nas intervenções, realização de procedimentos cirúrgicos e/ou adoecimento.

Foram considerados critérios de exclusão: 1) apresentar problemas de saúde que limitem ou impeçam a realização do programa de exercícios físicos; 2) apresentar condição associada a respostas inflamatórias sistêmicas, como gripe, enfermidades, procedimentos cirúrgicos recentes, e doenças do sistema imunológico; 3) ter frequência inferior a 65\% nas intervenções propostas; e, 4) não realizar todos os procedimentos de avaliação. O Comitê de Ética em Pesquisa da Universidade de Marília (UNIMAR), sob o protocolo $n^{\circ} 364$, aprovou a realização desta investigação.

Para evitar viés na coleta de dados, as avaliações e inquéritos foram aplicados por um único avaliador. A prevalência de doenças crônicas não transmissíveis da população em estudo foi obtida por meio do questionário de morbidades referidas, o qual foi complementado com informações sobre o uso de medicação e tempo sem menstruação. As variáveis do estudo foram mensuradas antes e após vinte semanas de intervenção.

O percentual de gordura corporal foi obtido pelo método de bioimpedância elétrica e estimado através de equação especifica para mulheres pós-menopausa ${ }^{13}$. O percentual de gordura maior ou igual a 33\% foi utilizado como ponto de corte para determinação de obesidade, conforme padrão de referência da Associação Brasileira para o Estudo da Obesidade e da Síndrome Metabólica ${ }^{11}$. Realizaram-se, ainda, medidas antropométricas de peso corporal e estatura, que foram utilizadas para o cálculo do índice de massa corporal (IMC), e de circunferência de cintura para determinação de da obesidade central.

A prática habitual de atividades físicas foi estimada pelo questionário de Baecke, com o propósito de confirmar a classificação das participantes quanto ao nível de atividade física, sendo consideradas sedentárias as participantes com menor que 150 minutos por semana de atividade física moderada ou vigorosa, nos últimos seis meses. ${ }^{12}$

Para a mensuração da pressão arterial sistólica (PAS) e diastólica (PAD) foi utilizado um esfigmomanômetro de coluna de mercúrio da (Marca Wan Med ${ }^{\circledR}$ ), com manguitos de tamanho apropriado a cada circunferência do braço do avaliado. As aferições foram realizadas no braço direito, respeitando-se repouso mínimo de cinco minutos. Todos os procedimentos metodológicos para aferição da pressão arterial (PA) seguiram as recomendações da VI Diretriz Brasileira de Hipertensão ${ }^{14}$.

Para coleta de sangue, todas as pacientes foram orientadas a se apresentarem para o exame em jejum mínimo de 12 horas, e evitar 
a prática de atividade física vigorosa e a ingestão de álcool nas 24 e 72 horas antecedentes à coleta sanguínea, respectivamente. Foram avaliados os parâmetros de glicemia, colesterol total (CT), lipoproteína de alta densidade $(H D L-c)$, lipoproteína de baixa densidade ( $L D L-c)$, e triglicérides (TG). A concentração plasmática de colesterol total e de triglicerídeos foi avaliada pelo método enzimático colorimétrico; de HDL-colesterol por inibição seletiva; e, análise de LDL-colesterol pela Equação de Friedewald para valores de triglicerídeos abaixo de 400 $\mathrm{mg} / \mathrm{dl}$. Os valores de glicose plasmática de jejum foram determinados pelo método glicose oxidase.

O Escore de Framingham foi utilizado para quantificar o efeito da intervenção sobre o risco de desenvolvimento de doença coronariana na próxima década, o qual foi calculado de acordo com as recomendações de D’Agostino et al. ${ }^{15}$

O programa de exercícios físicos foi estabelecido com base nas recomendações do Colégio Americano de Medicina do Esporte ${ }^{5,16}$, sendo este adaptado às condições disponíveis em Unidades Básicas de Saúde e Unidades de Saúde da Família ${ }^{17}$, e consistiu na oferta de exercícios cardiorrespiratórios e neuromusculares. O presente programa foi executado em vinte semanas, e suas atividades foram desenvolvidas em três sessões semanais de 90 minutos em dias alternados, totalizando 270 minutos de exercício físico por semana, onde cada sessão foi composta pelas seguintes fases: 1) inicial (10 minutos), destinada às avaliações de monitoramento e aquecimento; 2) principal (75 minutos), que compreende atividades aeróbias (50 minutos) e de força, resistência e alongamento muscular (25 minutos); e, 3) final (5 minutos), para as atividades de volta à calma.

O treinamento resistido foi realizado com exercícios isométricos e dinâmicos. O primeiro foi executado de quatro a seis exercícios em quatro séries, com 4 segundos de contração, seguidos por 10 a 30 segundos de recuperação, sendo a progressão da carga a cada 4 semanas com aumento do numero de exercícios ou redução da pausa. Foram realizados três a seis exercícios dinâmicos, executados sem sobrecarga adicional, em quatro séries, com 10 repetições e com 10 a 30 segundos de recuperação, sendo a progressão da carga a cada 4 semanas pelo aumento do número de exercícios e ou redução da pausa. As atividades de alongamento foram realizadas em todas as sessões, e foram compostas por 6 exercícios (dois para os membros inferiores, dois para membros superiores, um para região cervical e um para região lombar), sendo caracterizadas pela manutenção do alongamento no limiar da dor (2 séries de 30 segundos). Para as atividades de volta à calma realizaram-se atividades de relaxamento, de massagem ou de natureza cognitiva.

O consumo máximo de oxigênio $\left(\mathrm{VO}_{2 \max }\right)$ foi estimado pelo Protocolo de Rockport Walking Institute (caminhada de 1600 metros). A prescrição do trabalho aeróbio foi realizada em intensidade entre 50 e $65 \%$ do $\mathrm{VO}_{2}$ máximo, a partir do qual determinou-se a velocidade e distância da caminhada pela equação proposta nas Diretrizes do ACSM para Teste de Esforço e sua Prescrição ${ }^{18}$. A frequência cardíaca máxima ( $F C_{\max }$ foi determinada pela equação proposta por Gulati et al. ${ }^{19}$, para mulheres assintomáticas de 35 a 85 anos. Cada sessão de exercícios aeróbios tinha a duração de 50 minutos. Durante as sessões, a frequência cardíaca foi monitorada com o objetivo de verificar a relação entre $\% \mathrm{VO}_{2}$ máximo e zona alvo da frequência cardíaca de treinamento correspondente.

Os sujeitos do GnT foram orientados a manterem seus hábitos de vida. Foi realizado um encontro com os participantes deste grupo no início e ao final de vinte semanas para avaliação e reavaliação das variáveis citadas anteriormente. Ao final desse período, foram convidados a participar do programa de exercício físico.

\section{Análise Estatística}

Usando um desvio padrão médio igual a 3,8 e um erro de delineamento igual a 2,1, para testar uma diferença entre os valores médios do Escore de Framingham dos dois grupos, o poder calculado para o n amostral foi de $65 \%$. Os resultados foram organizados sob a forma de estatística descritiva, com valores de média e desvio padrão. A distribuição de normalidade foi verificada pelo teste de Kolmogorov-Smirnov com correção de Lillifors. As possíveis diferenças para os fatores de risco cardiovasculares e Escore de Framingham entre os grupos no início da intervenção foram identificadas pelo teste $t$ para amostras independentes, ou por seu similar não paramétrico, Mann-Whitney, quando o pressuposto de normalidade não foi atendido. Foi construída uma ANOVA para medidas repetidas com o objetivo de testar os efeitos de tempo, grupo e interação entre tempo e grupo. O teste $M$ de Box foi utilizado para verificar se as matrizes de covariâncias das variáveis dependentes observadas são iguais para os dois grupos e o Teste de Mauchly foi utilizado para testar a hipótese de esfericidade. No caso de rejeição da hipótese de esfericidade, as análises foram baseadas nos testes multivariados. Quando o efeito de interação foi estatisticamente significante, procedeu-se ao teste $t$ para amostras pareadas, com o objetivo de analisar o comportamento de cada grupo ao longo do tempo separadamente. Para todas as análises utilizou-se o software SPSS versão 19.0 for Windows, sendo adotado nível de significância de 5\%.

\section{RESULTADOS}

A amostra inicialmente foi classificada como sedentária, apresentando média de 41 \pm 58 minutos por semana de exercício físico moderado a intenso. O GT e GnT, não apresentaram diferenças significativas quanto à idade (anos) $(61,3 \pm 6,4$ vs. 59,8 $\pm 7,1 ; p=0,342)$, e tempo de menstruação (meses) (164,5 $\pm 93,2$ vs. 157,4 $\pm 108 ; p=0,768)$. Ao final do estudo o grupo treinado obteve aderência (\%) de participação nas intervenções de $77,1 \pm 10,4$ (\%), o que representou 208,4 $\pm 28,2$ min por semana de exercício físico moderado, com valor mínimo de 150 e máximo de 256 min por semana.

Quanto às doenças mais frequentes na amostra, nos grupo treinado e não treinado respectivamente, foram hipertensão arterial (74\% vs $66 \%$ ), dislipidemia (63 vs 54\%) e osteoporose (46 vs 43\%). As doenças menos frequentes forma artrite (29 vs 26\%), osteoartrite (17 vs 26\%) e diabetes tipo 2 (26 vs 20\%).

A tabela 1 descreve o comportamento das variáveis de composição

Tabela 1. Média土desvio-padrão (DP) das variáveis de composição corporal, nos momentos pré e pós-intervenção para o grupo treinado e não treinado.

\begin{tabular}{|c|c|c|c|c|}
\hline & \multicolumn{2}{|c|}{ Momento } & \multirow{2}{*}{$\begin{array}{c}\begin{array}{c}\text { Teste } t \\
\text { pareado }\end{array} \\
P\end{array}$} & \multirow{2}{*}{\begin{tabular}{|c|} 
Anova \\
medidas repetidas \\
Tempo vs. grupo \\
\end{tabular}} \\
\hline & Pré & Pós & & \\
\hline \multicolumn{5}{|l|}{$\mathrm{IMC}\left(\mathrm{kg} / \mathrm{m}^{2}\right)$} \\
\hline Treinado & $30,1 \pm 3,7^{a}$ & $29,3 \pm 3,7$ & $0,0001^{*}$ & \multirow{2}{*}{$0,001^{\#}$} \\
\hline Não Treinado & $33,4 \pm 5,9^{a}$ & $33,7 \pm 6,2$ & 0,215 & \\
\hline \multicolumn{5}{|l|}{$\mathrm{CC}(\mathrm{cm})$} \\
\hline Treinado & $93,3 \pm 10,3$ & $89,1 \pm 10,4$ & $0,0001^{* *}$ & \multirow{2}{*}{$0,013^{\#}$} \\
\hline Não Treinado & $98,0 \pm 13,7$ & $97,1 \pm 13,5$ & 0,377 & \\
\hline \multicolumn{5}{|l|}{$\%$ Gordura } \\
\hline Treinado & $54,5 \pm 2,9$ & $53,2 \pm 3,3$ & $0,001^{*}$ & \multirow{2}{*}{$0,001^{\#}$} \\
\hline Não Treinado & $55,0 \pm 4,0$ & $57,0 \pm 3,8$ & $0,0001^{*}$ & \\
\hline \multicolumn{5}{|l|}{$\%$ Massa magra } \\
\hline Treinado & $45,4 \pm 2,9$ & $54,5 \pm 2,9$ & $0,001^{*}$ & \multirow{2}{*}{$0,001^{\#}$} \\
\hline Não Treinado & $45,0 \pm 4,0$ & $42,9 \pm 3,8$ & $0,0001^{*}$ & \\
\hline
\end{tabular}

Nota: IMC índice de massa corporal; CC circunferência de cintura. ${ }^{a}$ indica diferenças significativas entre os grupos antes do início da intervençăo. * diferença significativa dentro do grupo pelo teste t para amostras pareadas; *** diferença significativa dentro do grupo pelo teste de Wilcoxon; \# interação grupo versus momento pelo teste de Anova de medidas repetidas. 
corporal para os grupos entre os momentos pré e pós-intervenção. Na avaliação inicial foi observada diferença significante entre os grupos, apenas para a variável IMC. No GT observou-se redução significativa do IMC, circunferência de cintura e percentual de gordura, com também aumento do percentual da massa magra. No GnT, não foram detectadas alterações nos valores de IMC e circunferência de cintura, com aumento significativo na percentagem de gordura e redução no percentual de massa magra.

O comportamento da glicemia de jejum e perfil lipídico para os grupos, nos momentos pré e pós-intervenção, são apresentadas na tabela 2. Não foram observadas diferenças significativas entre os grupos antes do início da intervenção. No GT observou-se reduções significativas do triglicerídeos e VLDL-c após a intervenção. O GnT apresentou aumento significativo da glicemia de jejum e redução dos valores de HDL-c, enquanto o GT não demonstrou variações significantes no HDL-c e glicemia de jejum, mantendo os valores dentro do padrão de normalidade. Os valores de colesterol total e LDL-c não apresentaram mudanças significativas em ambos os grupo, nem interação.

Os valores do Escore de Framingham, frequência cardíaca de repouso $(\mathrm{FCr})$, consumo máximo de oxigênio $\left(\mathrm{VO}_{2 \mathrm{max}}\right)$, pressão arterial sistólica e diastólica para os grupos, nos momentos pré e pós-intervenção, são apresentadas na tabela 3. Não foram observadas diferenças significativas entre os grupos antes da intervenção. O GT apresentou reduções significantes nos valores de FC de respouso, PAS e Escore de Framingham, além de aumento nos valores de $\mathrm{VO}_{2 \max }$. O GnT apresentou aumento singinificativo nos valores de $\mathrm{VO}_{2 \max }$ PAS e Escore de Framingham.

\section{DISCUSSÃO}

Os resultados observados indicam que o modelo de intervenção com exercícios físicos proposto no estudo tem efeito positivo sobre a composição corporal, pressão arterial sistólica, a maior parte dos parâmetros metabólicos e o Escore de Franmingham. Em contrapartida, a não exposição a esta intervenção acarretou na piora de vários dos parâmetros supracitados.

No que diz respeito à composição corporal e às variáveis hemodi-

Tabela 2. Média e desvio-padrão (DP) da glicemia de jejum e perfil lipídico, nos momentos pré e pós-intervenção para o grupo treinado (GT) e não treinado (GnT).

\begin{tabular}{|c|c|c|c|c|}
\hline & \multicolumn{2}{|c|}{ Momento } & \multirow{2}{*}{$\begin{array}{c}\begin{array}{c}\text { Teste } t \\
\text { pareado }\end{array} \\
P \\
\end{array}$} & \multirow{2}{*}{\begin{tabular}{|c|}
$\begin{array}{c}\text { Anova medidas } \\
\text { repetidas }\end{array}$ \\
Tempo vs. grupo \\
\end{tabular}} \\
\hline & Pré & Pós & & \\
\hline \multicolumn{5}{|c|}{ Glicemia de jejum $(\mathrm{mg} / \mathrm{dL})$} \\
\hline Treinado & $96,7 \pm 18,5$ & $95,6 \pm 15,2$ & 0,706 & \multirow{2}{*}{$0,0001^{\#}$} \\
\hline Não Treinado & $95,2 \pm 18,4$ & $113,7 \pm 28,8$ & $0,0001^{* *}$ & \\
\hline \multicolumn{5}{|c|}{ Colesterol total (mg/dL) } \\
\hline Treinado & $207,2 \pm 35,6$ & $206,2 \pm 34,2$ & 0,844 & \multirow{2}{*}{0,205} \\
\hline Não Treinado & $212,4 \pm 30,3$ & $219,6 \pm 36,5$ & 0,194 & \\
\hline \multicolumn{5}{|c|}{ Triglicerídeos (mg/dL) } \\
\hline Treinado & $148,4 \pm 66,1$ & $122,8 \pm 40,7$ & $0,006^{* *}$ & \multirow{2}{*}{$0,006^{\#}$} \\
\hline Não Treinado & $146,0 \pm 63,4$ & $155,7 \pm 65,3$ & 0,258 & \\
\hline \multicolumn{5}{|c|}{ HDL colesterol (mg/dL) } \\
\hline Treinado & $51,9 \pm 12,9$ & $51,8 \pm 12,3$ & 0,952 & 0,121 \\
\hline Não Treinado & $55,17 \pm 10,5$ & $51,7 \pm 11,0$ & $0,017^{* *}$ & \\
\hline \multicolumn{5}{|c|}{ LDL colesterol (mg/dL) } \\
\hline Treinado & $130,0 \pm 30,2$ & $129,8 \pm 29,7$ & 0,967 & \multirow{2}{*}{0,180} \\
\hline Não Treinado & $128,0 \pm 30,8$ & $136,7 \pm 33,0$ & 0,131 & \\
\hline \multicolumn{5}{|c|}{ VLDL colesterol (mg/dL) } \\
\hline Treinado & $29,7 \pm 13,2$ & $24,5 \pm 8,0$ & $0,005^{* *}$ & \multirow{2}{*}{$0,005^{\#}$} \\
\hline Não Treinado & $29,2 \pm 12,6$ & $31,2 \pm 13,1$ & 0,236 & \\
\hline
\end{tabular}

Nota: ${ }^{~}$ indica diferenças significativas entre os grupos antes do início da intervençăo. ${ }^{*}$ diferença significativa dentro do grupo pelo teste t para amostras pareadas; ${ }^{* *}$ diferença significativa dentro do grupo pelo teste de Wilcoxon. \# interação grupo versus momento pelo teste de Anova de medidas repetidas.
Tabela 3. Média e desvio-padrão (DP) da pressão arterial e Escore de Framingham, nos momentos pré e pós-intervenção para o grupo treinado (GT) e não treinado (GnT). Análise do efeito terapêutico da intervenção.

\begin{tabular}{|c|c|c|c|c|}
\hline & \multicolumn{2}{|c|}{ Momento } & \multirow{2}{*}{$\begin{array}{c}\begin{array}{c}\text { Teste } t \\
\text { pareado }\end{array} \\
P\end{array}$} & \multirow{2}{*}{$\begin{array}{c}\text { Anova medidas } \\
\text { repetidas }\end{array}$} \\
\hline & Pré & Pós & & \\
\hline \multicolumn{5}{|c|}{ FC repouso (bpm) } \\
\hline Treinado & $72,8 \pm 9,0$ & $65,8 \pm 5,7$ & $0,0001^{*}$ & \multirow{2}{*}{$0,001^{\#}$} \\
\hline Não Treinado & $73,7 \pm 7,3$ & $73,3 \pm 8,3$ & 0,790 & \\
\hline \multicolumn{5}{|c|}{$\mathrm{VO}_{2 \max }(\mathrm{ml} / \mathrm{kg} / \mathrm{min})$} \\
\hline Treinado & $18,0 \pm 4,3$ & $22,3 \pm 5,3$ & $0,0001^{*}$ & \multirow{2}{*}{$0,009^{\#}$} \\
\hline Não Treinado & $16,4 \pm 3,5$ & $18,3 \pm 4,8$ & $0,004^{*}$ & \\
\hline \multicolumn{5}{|l|}{ PAS (mmHg) } \\
\hline Treinado & $128,0 \pm 14,6$ & $119,2 \pm 10,3$ & $0,0001^{*}$ & \multirow{2}{*}{$0,0001^{\#}$} \\
\hline Não Treinado & $128,6 \pm 10,5$ & $133,8 \pm 12,0$ & $0,025^{*}$ & \\
\hline \multicolumn{5}{|l|}{ PAD (mmhg) } \\
\hline Treinado & $79,9 \pm 9,9$ & $78,8 \pm 7,0$ & 0,504 & \multirow{2}{*}{0,553} \\
\hline Não Treinado & $83,9 \pm 7,3$ & $84,1 \pm 9,8$ & 0,941 & \\
\hline \multicolumn{5}{|c|}{ Escore de Framingham } \\
\hline Treinado & $13,08 \pm 4,01$ & $11,77 \pm 4,13$ & $0,010^{*}$ & \multirow{2}{*}{$0,001^{\#}$} \\
\hline Não Treinado & $12,82 \pm 3,27$ & $13,91 \pm 4,02$ & $0,043^{*}$ & \\
\hline
\end{tabular}

Nota: $F C$ frequência cardíaca de repouso; $\mathrm{VO}_{2 \operatorname{ma}}$ consumo máximo de oxigênio; PAS pressão arterial sistólica; PAD pressão arterial diastólica. A letra "a"sobrescrita indica diferenças significativas entre os grupos antes do início da inter venção. * diferença significativa dentro do grupo pelo teste t para amostras pareadas; ** diferença significativa dentro do grupo pelo teste de Wilcoxon. \# interação grupo versus momento pelo teste de Anova de medidas repetidas.

nâmicas em mulheres na pós-menopausa acometidas por alterações metabólicas, vários são os estudos que encontraram efeito benéfico do exercício físico ${ }^{17,18,20-22}$. Verificou-se que a redução dos valores antropométricos aconteceu tanto após a realização de exercícios de caminhada moderada por doze ${ }^{21}$ ou dezesseis semanas ${ }^{17}$, como após a realização de programas com exercícios combinados (aeróbios e de força) durante longos ${ }^{20}$ e curtos ${ }^{21}$ períodos, corroborando com os achados deste estudo.

Os exercícios físicos propostos no presente estudo foram suficientes para melhorar os valores da pressão arterial sistólica (PAS), sem alterar a diastólica. Resultados semelhantes também foram identificados em mulheres na menopausa com síndrome metabólica, submetidas a 16 semanas de caminhadas ${ }^{17}$, e em homens e mulheres com síndrome metabólica, submetidos a nove meses de exercícios aeróbios em cicloergômetros combinados com exercícios de força ${ }^{20}$.

Em contrapartida, Bateman et al. ${ }^{18}$ verificaram reduções somente nos valores de pressão arterial diastólica (PAD) após 16 semanas de exercícios físicos aeróbios combinados ao de força; este fato pode estar relacionado à faixa etária dos participantes do estudo, que possuíam entre 18 e 70 anos. Entretanto, as mulheres participantes do estudo de Choi et al. ${ }^{21}$ apresentaram melhoras tanto na PAS quanto na PAD após 12 semanas de caminhada; este foi o único protocolo que ofertava atividade física cinco vezes por semana, sendo que a frequência semanal de exposição pode ter sido o diferencial para os resultados encontrados.

Quanto aos parâmetros metabólicos, verificou-se que os resultados obtidos nos estudos são distintos. Na presente pesquisa, a glicemia do GT não se alterou significativamente após a intervenção com exercícios, o que também foi observado em outros estudos com protocolos de exercícios semelhantes ${ }^{18,20,22}$. Observou-se, que em todos estes trabalhos os valores iniciais de glicemia encontravam-se dentro dos padrões de normalidade, o que pode ter contribuído para a ausência de reduções nos parâmetros glicêmicos.

Todavia, apesar do protocolo de exercício utilizado não ter modificado os valores de glicemia entre os períodos pré e pós-intervenção, ele impediu que houvesse aumento significante deste parâmetro, contrariamente ao observado nos pacientes do GnT e, portanto, pode-se afirmar que o treinamento exerceu um efeito positivo sobre esta variável, contribuindo para manutenção do valores dentro do padrão de normalidade. Estudos realizados em mulheres com diabetes tipo 2 demostraram que a oferta de caminhada ${ }^{21}$ ou sua associação com trei- 
namento resistido ${ }^{23}$ são eficientes para reduzir ou manter parâmetros glicêmicos normais após 12 e 16 semanas, respectivamente.

Quanto ao perfil lipídico, verificou-se que a intervenção proposta neste estudo foi eficiente em reduzir os valores de triglicérides e VLDL-c, apresentando ainda efeito positivo no $\mathrm{HDL}$-c, uma vez que impediu a redução deste parâmetro no GT, diferente do observado no GnT. Apesar deste assunto ser muito explorado na literatura, observa-se que os efeitos dos exercícios físicos sobre o perfil lipídico ainda se mantém bastante controversos, uma vez que há estudos que apontam tanto a ausência de efeitos em todos os parâmetros ${ }^{22,24}$, como a melhora de todos $^{19}$ ou de apenas alguns deles ${ }^{18,20,23}$.

Nos trabalhos onde se constatou melhora do perfil lipídico pelo exercício físico, o triglicérides foi o que sofreu alteração significativa em todos os protocolos de intervenção realizados ${ }^{17,18,20,23}$, inclusive o do presente estudo, indicando que esta variável seja mais suscetível ao efeito do treinamento físico supervisionado. Em seguida, observou-se maior frequência de alterações no HDL-c $\mathrm{C}^{17,20,23}$ e no LDL-c ${ }^{17,20}$, ao passo que no colesterol total apenas dois estudos apresentaram alterações estatisticamente significativas neste parâmetro ${ }^{17,24}$.

No estudo de Petković-Košcal et al. ${ }^{24}$, desenvolvido para analisar o efeito temporal da intervenção com exercício físico sobre o perfil lipídico de pacientes intolerantes à glicose (faixa etária de 30 a 60 anos), verificou-se que efeitos significantes no perfil lipídico são tempo dependentes e somente ocorrem após 12 meses de intervenção. Não obstante, tanto o presente estudo como em outros foi encontrada redução de parâmetros lipídicos com tempo de intervenção inferior a 12 meses, indicando que outros aspectos, além dos associados ao tempo, podem estar relacionados com alterações efetivas no perfil lipídico 17,18,20,23.

Às alterações de colesterol total e LDL-c, Ahmed et al. ${ }^{25}$, aponta que o principal efeito do exercício físico regular não é sobre a redução das concentrações destes parâmetros, mas no aumento do tamanho da partícula de LDL-c tornando-a menos densa. Dessa forma, sua ação acarretaria em alterações qualitativas e não quantitativas de alguns parâmetros lipídicos o que, em parte, pode contribuir para a redução

\section{REFERÊNCIAS}

1. Eshtiaghi R, Esteghamati A, Nakhjavani M. Menopause is an independent predictor of metabolic syndrome in Iranian women. Maturitas. 2012;65(3):262-6.

2. Oliveira A, Mancini Filho J. Perfil nutricional e lipídico de mulheres na pós-menopausa com doença arterial coronariana. Arq Bras Cardiol. 2005;84(4):325-9.

3. Orsatti FL, Nahas EAP, Nahas-Neto J, Maestá N, Padoani NP, Orsatti, C.L. Indicadores antropométricos e doenças crônicas não transmissíveis em mulheres na pós-menopausa da região Sudeste do Brasil. Rev Bras Ginecol Obstet. 2008;30(4):182-9.

4. Haslam DW, James WP. Obesity. Lancet 2005;366(9492):1197-209.

5. Haskell WL, Lee IM, Pate RR, Powell KE, Blair SN, Franklin BA, et al. Physical activity and public health: Updated recommendation for adults from the American college of sports medicine and the American heart association. Med Sci Sports Exerc. 2007;39(8):1423-34.

6. Gremeaux V1, Gayda M, Lepers R, Sosner P, Juneau M, Nigam A. exercise and longevity. Maturitas. 2012;73(4):312-7.

7. Kokobum E, Luciano E, Sibuya CY, Queiroga MR, Ribeiro PAB, Silveira RF, et al. Programa de atividade física em unidades básicas de saúde: relato de experiência no município de Rio Claro-SP. Rev Bras Ativ Fís Saúde. 2007;12(1):45-53.

8. Monteiro HL, Rolim LMC, Squinca DM, Silva FC, Ticianeli CCC, Amaral SL. Efetividade de um programa de exercício físico no condicionamento físico, perfil metabólico e pressão arterial de pacientes hipertensos. Rev Bras Med Esporte. 2007;13(2):107-2.

9. Malta DC, Silva JB. Policies to promote physical activity in Brazil. Lancet. 2012;380(9838):195-6.

10. Bonfim MR, Costa JL, Monteiro HL. Ações de educação física na saúde coletiva brasileira: expectativas versus evidências. Rev Bras Ativ Fís Saúde. 2012;17(3):167-73.

11. Associação Brasileira para o Estudo da Obesidade e da Síndrome Metabólica. Diretrizes Brasileiras de obesidade. 2009/2010 / ABESO. 3a. ed. Itapevi, SP: AC Farmacêutica; 2009.

12. Alves JGB, Siqueira FV, Figueiroa JN, Facchini LA, Silveira DS, Piccini RX, et al. Prevalência de adultos e idosos insuficientemente ativos moradores em áreas de unidades básicas de saúde com e sem Programa Saúde da Família em Pernambuco, Brasil. Cad Saúde Pública. 2010;26(3):543-56.

13. Kanellakis S, Kourlaba G, Moschonis G, Vandouro A, Manios Y. Development and validation of two equations estimating body composition for overweight and obese postmenopausal women. Maturitas. 2010;65(1):64-8

14. Sociedade Brasileira de Cardiologia, Sociedade Brasileira de Hipertensão, Sociedade Brasileira de Nefrologia. VI Diretrizes Brasileiras de hipertensão. Arq Bras Cardiol. 2010; 95(Supl 1):1-51.

15. D'Agostino RB, Vasan RS, Pencina MJ, Wolf PA, Cobain M, Massaro JM, et al. General cardiovascular risk profile for use primary care: The Framingham Heart Study. Circulation 2008;117(6):743-53.

16. Garber CE, Blissmer B, Deschenes MR, Franklin BA, Lamonte MJ, Lee IM. American College of Sports Medicine position stand. Quantity and quality of exercise for developing and maintaining cardiorespi- do risco de doença arterial coronariana (DAC).

Verificou-se redução significante no escore de Framingham em resposta ao protocolo de exercícios físicos no GT, o que indica menor risco para desenvolvimento de doenças coronarianas, como também interação entre grupo e tempo de intervenção, indicando uma tendência do GnT a aumentar o risco de desenvolvimento destas doenças. De modo semelhante, outros estudos verificaram reduções significativas do escore de Framingham após a realização de exercício físico ${ }^{26-28}$, embora não tenhamos encontrado na literatura nenhum estudo com casuística semelhante a de nosso estudo, tanto em relação a população de estudo, quanto ao ambiente de realização do programa de exercício físico.

Fatores como o tempo de intervenção, a intensidade da sobrecarga de exercício físico e a adequação dos valores iniciais dos parâmetros metabólicos, podem modificar as respostas dos fatores de risco, em especial o perfil lipídico, frente a realização de programas de exercícios físicos para mulheres pós-menopausa. Atualmente, profissionais de saúde continuam a divergir fortemente sobre o tipo específico, padrão, quantidade e intensidade de exercícios físicos que podem proporcionar benefícios mensuráveis à saúde ${ }^{29}$.

\section{CONCLUSÃO}

Verificamos que mesmo adaptando as recomendações de exercício físico para a realidade da USF, caracterizada por espaço reduzido e pouca disponibilidade de materiais, a prescrição de exercícios físicos por métodos indiretos foi suficiente para melhorar o estado de saúde de mulheres obesas atendidas pelo sistema único de saúde, especificamente reduzindo a gordura corporal, a pressão arterial sistólica, os valores de triglicerídeos e VLDL-c, bem como o Escore de Framingham, sendo ainda relacionado a efeitos benéficos no controle da glicemia de jejum e no HDL-c.

Todos os autores declararam não haver qualquer potencial conflito de interesses referente a este artigo. ratory, musculoskeletal, and neuromotor fitness in apparently healthy adults: guidance for prescribing exercise. Med Sci Sports Exerc. 2011;43(7):1334-59.

17. Roussel M, Garnier S, Lemoine S, Gaubert I, Charbonnier L, Auneau G, et al. Influence of a walking program on the metabolic risk profile of obese postmenopausal women. Menopause. 2009;16(3):566-75.

18. Bateman LA, Slentz CA Willis LH, Shields AT, Piner LW Bales CW et al. Comparison of aerobic versus resistance exercise training effects on metabolic syndrome (from the Studies of a Targeted Risk Reduction Intervention Through Defined Exercise -STRRIDE-AT/RT). Am J Cardiol. 2011;108(6):838-44.

19. Gulati M, Shaw $L$, Thisted RA, Black HR, Merz CNB, Arnsdorf MF. Heart rate response to exercise stress testing in asymptomatic women: the St. James women take heart project. Circulation 2010;122(2):130-7.

20. Gremeaux V, Drigny J, Nigam A, Juneau M, Guilbeault V, Latour E, et al. Long-term lifestyle intervention with optimized high-intensity interval training improves body composition, cardiometabolic risk, and exercise parameters in patients with abdominal obesity. Am J Phys Med Rehabil. 2012;91(11):941-50.

21. Choi MK, Han KA, Ahn HJ, Hwang SY, Hong HC, Choi HY, et al. Effects of exercise on sRAGE levels and cardiometabolic risk factors in patients with type 2 diabetes: a randomized controlled trial. J Clin Endocrinol Metab. 2012; 97(10):3751-8.

22. Choquette S, Riesco E, Cormier E, Dion T, Aubertin-Leheudre M, Dionne IJ. Effects of soya isoflavones and exercise on body composition and clinical risk factors of cardiovascular diseases in overweight postmenopausal women: a 6-month double-blind controlled trial. Br J Nutr. 2011;105(8):1199-209.

23. Chirsto ZE, Tokmakidis SP, Volaklis KA, Kotsa K, Touvra AM, Douda E, et al. Lipoprotein profile, glycemic control and physical Wtness after strength and aerobic training in post-menopausal women with type 2 diabetes. Eur J Appl Physiol. 2009;106(6):901-7.

24. Petković-Košćal M, Damjanov V, Djonović N. Influence of moderate physical activity on the level of plasma lipoproteins in subjects with impaired glucose tolerance. Srp Arh Celok Lek. 2012;140(1-2):51-7.

25. Ahmed HM, Blaha MJ, Nasir K, Rivera JJ, Blumenthal RS. Effects of Physical Activity on Cardiovascular Disease. Am J Cardiol. 2012;109(2):288-95.

26. Marcon ER, Gus I, Neumann CR. Impacto de um programa mínimo de exercícios físicos supervisionados no risco cardiometabólico de pacientes com obesidade mórbida. Arq Bras Endocrinol Metab. 2011;55:331-8.

27. Dutheil F, Lac G, Lesourd B, Chapier R, Walther G, Vinet A. Different modalities of exercise to reduce visceral fat mass and cardiovascular risk in metabolic syndrome: the RESOLVE* randomized Trial. Int J Cardiol. 2013;168(4):3634-42

28. Gidlow CJ, Cochrane T, Davey R, Beloe M, Chambers R, Kumar J, et al. One-year cardiovascular risk and quality life changes in participants of a health trainer service. Perspect Public Health. 2014:134(3):135-44.

29. Ross R, Hudson R, Day AG, Lam M. Dose-response effects of exercise on abdominal obesity and risk factors for cardiovascular disease in adults: Study rationale, design and methods. Contemp Clin Trials. 2013;34(1):155-60. 